\title{
A atuação do médico residente em ortopedia e traumatologia em um hospital de grande porte do Sistema Único de Saúde: Qual a visão do usuário?*
}

\section{The Role of the Resident Physician in Orthopedics and Traumatology in a Large Hospital of the Unified Health System: What is the User's view?}

Luiz Felippe Mokdeci Martins de Oliveira1® Daniel Alves Ramallo ${ }^{1,2} \quad$ João Victor Silveira Möller2® Ana Carolina Leal ${ }^{3}$ Gabriel Araujo Ribeiro ${ }^{20}$ joão Antonio Matheus Guimarães ${ }^{20}$

${ }^{1}$ Centro de Atenção Especializada em Cirurgia da Coluna Vertebral, Instituto Nacional de Traumatologia e Ortopedia Jamil Haddad, Rio de Janeiro, RJ, Brasil

${ }^{2}$ Centro de Atenção Especializada em Trauma Ortopédico, Instituto Nacional de Traumatologia e Ortopedia Jamil Haddad, Rio de Janeiro, RJ, Brasil

${ }^{3}$ Divisão de Ensino e Pesquisa, Instituto Nacional de Traumatologia e Ortopedia Jamil Haddad, Rio de Janeiro, RJ, Brasil
Endereço para correspondência João Antonio Matheus Guimarães, MSc, PhD, Divisão de Ensino e pesquisa, Instituto Nacional de Traumatologia e Ortopedia Jamil Haddad, Av. Brasil, 500, São Cristovão, Rio de Janeiro, RJ, 20940-070, Brasil

(e-mail: jmatheusguimaraes@gmail.com).

Rev Bras Ortop 2021;56(4):438-445.

\section{Resumo}

Palavras-chave

- médico residente

- ortopedia

- traumatologia

- cirurgia ortopédica
Objetivo Avaliar o conhecimento de pacientes atendidos em um hospital-escola acerca da formação acadêmica e profissional do médico residente em ortopedia e traumatologia, bem como sua área de atuação, e determinar a percepção de conforto e segurança do paciente em relação a ser assistido pelo médico residente em diferentes etapas do tratamento.

Métodos Foi realizado um estudo transversal com pacientes internados em um hospital de ortopedia de grande porte do Sistema Único de Saúde (SUS). Os dados foram coletados a partir da aplicação de um questionário contendo 19 questões objetivas que avaliaram parâmetros sociodemográficos e a percepção do paciente quanto à atuação do residente. Os dados foram analisados de forma a avaliar a frequência das respostas obtidas.

Resultados Foram avaliados 152 participantes, predominantemente do sexo masculino $(62,5 \%)$ e com idade entre 36 e 55 anos (41,3\%). Apenas 43,3\% tinham conhecimento sobre a formação acadêmica do residente. Os pacientes relataram se

\footnotetext{
Trabalho desenvolvido no Instituto Nacional de Traumatologia e Ortopedia Jamil Haddad, Rio de Janeiro, Brasil.
}

recebido

29 de Abril de 2020

aceito

06 de Julho de 2020

Publicado on-line

Dezembro 16, 2020
DOI https://doi.org/ $10.1055 / \mathrm{s}-0040-1718513$ ISSN 0102-3616. (c) 2020. Sociedade Brasileira de Ortopedia e Traumatologia. All rights reserved.

This is an open access article published by Thieme under the terms of the Creative Commons Attribution-NonDerivative-NonCommercial-License, permitting copying and reproduction so long as the original work is given appropriate credit. Contents may not be used for commercial purposes, or adapted, remixed, transformed or built upon. (https://creativecommons.org/ licenses/by-nc-nd/4.0/)

Thieme Revinter Publicações Ltda., Rua do Matoso 170, Rio de Janeiro, RJ, CEP 20270-135, Brazil 


\begin{abstract}
Keywords

- resident doctor

- orthopedics and traumatology

- orthopedic surgery

Objective To assess the knowledge of patients seen at a teaching hospital about the academic and professional training of the resident doctor in orthopedics and traumatology, as well as his area of expertise, and determine the perception of the patients of comfort and safety in relation to being assisted by the resident doctor at different stages of treatment.

Methods A cross-sectional study was conducted with patients admitted to a large orthopedics hospital of the Brazilian Unified Health System (SUS, in the Portuguese acronym). Data were collected through the application of a questionnaire containing 19 objective questions that assessed sociodemographic parameters and the perception of the patient of the performance of the resident. The data were analyzed to assess the frequency of responses obtained.

Results 152 participants were evaluated, predominantly male (62.6\%) and aged between 36 and 55 years old (41.3\%). Only $43.3 \%$ were aware of the academic background of the resident. Patients reported feeling safer and more comfortable being assisted by the doctor together with the resident in the outpatient consultation (43.3\%), in the nursing ward (39.3\%) and during surgery (61\%). As for the performance of the resident, $80.2 \%$ stated that the resident doctor improves communication between the patient and the main surgeon; however, only $11 \%$ said they would feel safe and comfortable being cared for exclusively by residents in the surgical environment, if allowed.

Conclusion The participation of resident physicians in the care is well received by the patients if they are in the company of the attending physician. Patients identify residents as a facilitating bridge in the communication with attending physicians.
\end{abstract}

sentir mais seguros e confortáveis em serem assistidos pelo médico em conjunto com o residente na consulta ambulatorial (43,3\%), na enfermaria (39,3\%) e durante a cirurgia (61\%). Quanto à atuação do residente, $80,2 \%$ afirmaram que o médico residente melhora a comunicação entre o paciente e o cirurgião principal, entretanto e apenas $11 \%$ disseram se sentir seguros e confortáveis sendo cuidados exclusivamente por residentes no ambiente cirúrgico, caso fosse permitido.

Conclusão A participação de médicos residentes nos cuidados é bem recebida pelos pacientes, desde que em companhia do médico assistente. Os pacientes identificam nos residentes uma ponte facilitadora na comunicação com os médicos assistentes.

\section{Introdução}

Nos últimos anos, a prática de sobreposição de cirurgias, que consiste em um cirurgião principal coordenar duas ou mais salas de cirurgia ao mesmo tempo, vem sendo alvo de extensos debates entre a comunidade médica e a sociedade, ${ }^{1-3}$ além de ganhar cada vez mais espaço nos principais periódicos ao redor do mundo. ${ }^{4-8}$ Esta prática, apesar de não ser legalmente permitida no Brasil, é bastante comum em centros médicos acadêmicos, sendo essencial para o treinamento de residentes e apresentando benefícios como a redução do tempo de espera dos pacientes para a realização de cirurgia, diminuição dos custos cirúrgicos, otimização da receita dos hospitais, além de propiciar o desenvolvimento de habilidade e autonomia dos médicos residentes e aumentar o número de médicos envolvidos nos cuidados aos pacientes. $^{3,5}$
Ainda que sejam necessárias pesquisas mais extensas sobre este tema, estudos sugerem não haver um aumento dos riscos e complicações envolvendo tal prática. ${ }^{3,9}$ Foi encontrada uma diminuição das taxas de complicações gerais e nenhum impacto nas complicações de feridas operatórias em cirurgias com a participação de médicos residentes de ortopedia e traumatologia, apesar dos tempos cirúrgicos prolongados. ${ }^{10}$

Entretanto, embora alguns estudos tenham abordado o impacto da participação de residentes nas cirurgias e nos desfechos do procedimento, incluindo em cirurgias sobrepostas, ${ }^{8,11}$ poucos trabalhos investigaram a percepção do paciente sobre a participação do médico residente em seu tratamento. ${ }^{12}$ Dessa forma, o propósito do presente estudo é identificar o conhecimento dos pacientes acerca da formação acadêmica e profissional do médico residente de ortopedia e 
traumatologia, bem como avaliar a percepção desses pacientes em relação aos cuidados dos residentes em ambiente cirúrgico, ambulatorial e enfermarias.

\section{Materiais e Métodos}

\section{Desenho do Estudo}

Foi realizado um estudo transversal, baseado em uma amostragem por conveniência consecutiva, incluindo pacientes internados em um hospital de grande porte do Sistema Único de Saúde (SUS) no período entre maio de 2019 a julho de 2019. O presente estudo foi aprovado pelo Comitê de Ética e Pesquisa da Instituição (CAAE. 12159819.9.0000.5273)

\section{População do Estudo}

Foram incluídos pacientes de ambos os gêneros, com idade $>18$ anos e alfabetizados, que se encontravam em internação pré-operatória para tratamento de lesões traumáticas ortopédicas. Foram excluídos pacientes sedados, comatosos e com déficit cognitivo, bem como os que se recusaram ao preenchimento do Termo de Consentimento Livre e Esclarecido (TCLE).

\section{Elaboração e Aplicação do Questionário}

Para a realização do estudo, foi elaborado um questionário, contendo 19 perguntas objetivas, abordando questões relativas aos aspectos sociodemográficos dos participantes, ao conhecimento sobre a formação acadêmica do residente e sobre a percepção acerca da participação do residente em seu tratamento (-Anexo 1).

O estudo foi realizado em uma única fase, na qual a amostra foi submetida a uma análise transversal por meio da aplicação do questionário durante a internação.

\section{Análise dos Resultados}

Para as análises, foram determinadas as frequências de respostas e a correlação entre as variáveis sociodemográficas e perguntas selecionadas. As análises estatísticas foram realizadas utilizando-se o programa GraphPad Prism versão 7.0 (GraphPad Software, San Diego, CA, EUA). O teste de quiquadrado foi utilizado para verificar possíveis associações entre variáveis categóricas e, quando necessário, foi utilizado o teste exato de Fisher. Um valor de p de 0,05 foi considerado significativo.

\section{Resultados}

Foram incluídos 152 pacientes que se encontravam em internação pré-operatória para cirurgia de trauma ortopédico. As características sociodemográficas da amostra estão apresentadas na - Tabela 1. A maior parte dos participantes era do sexo masculino $(62,6 \%, \mathrm{n}=95)$, com idade entre 36 e 55 anos $(41,3 \%, n=62)$, com ensino médio completo (30,9\% $\mathrm{n}=47$ ) e declararam ter renda familiar mensal de até $\mathrm{R}$ $\$ 1.499,99(68,4 \%, n=91)$. Apenas $5,3 \%(n=8)$ dos participantes trabalhavam como profissionais da área da saúde, e $30 \%$ deles $(n=45)$ possuíam parentesco direto com profissionais da área da saúde.
Tabela 1 Características sociodemográficas da amostra

\begin{tabular}{|c|c|}
\hline Respostas & $\mathrm{N}(\%)$ \\
\hline \multicolumn{2}{|l|}{ Idade } \\
\hline 18 a 35 anos & $45(30 \%)$ \\
\hline 36 a 55 anos & $62(41,3 \%)$ \\
\hline 56 a 75 anos & $33(22 \%)$ \\
\hline$>76$ anos & $10(6,6 \%)$ \\
\hline \multicolumn{2}{|l|}{ Gênero } \\
\hline Masculino & $95(62,5 \%)$ \\
\hline Feminino & $57(37,5 \%)$ \\
\hline \multicolumn{2}{|l|}{ Escolaridade } \\
\hline Ensino fundamental incompleto & $44(29,8 \%)$ \\
\hline Ensino fundamental completo & $17(11,1 \%)$ \\
\hline Ensino médio incompleto & $33(21,7 \%)$ \\
\hline Ensino médio completo & $47(30,9 \%)$ \\
\hline Ensino superior incompleto & $5(3,2 \%)$ \\
\hline Ensino superior completo & $6(3,9 \%)$ \\
\hline \multicolumn{2}{|l|}{ Renda mensal } \\
\hline até $R \$ 1.499,99$ & $91(68,4 \%)$ \\
\hline De $R \$ 1.500$ a $R \$ 2.999$ & $26(19,5 \%)$ \\
\hline De $R \$ 3.000$ a $R \$ 4.999$ & $7(5,2 \%)$ \\
\hline De $R \$ 5.000$ a $R \$ 9.999$ & $7(5,2 \%)$ \\
\hline$>\mathrm{R} \$ 10.000$ & $2(1,5 \%)$ \\
\hline \multicolumn{2}{|l|}{ Trabalha como profissional da saúde } \\
\hline $\operatorname{Sim}$ & $8(5,3 \%)$ \\
\hline Não & $143(94,7 \%)$ \\
\hline \multicolumn{2}{|l|}{$\begin{array}{l}\text { Possui parente de primeiro grau que } \\
\text { trabalha como profissional da saúde }\end{array}$} \\
\hline Sim & $45(30 \%)$ \\
\hline Não & $105(70 \%)$ \\
\hline \multicolumn{2}{|c|}{ Submetido a procedimento cirúrgico prévio } \\
\hline $\operatorname{Sim}$ & $109(72,2 \%)$ \\
\hline Não & $42(27,8 \%)$ \\
\hline
\end{tabular}

Quanto às expectativas dos pacientes, a maioria relatou que esperava encontrar tanto o médico principal (72\%, $\mathrm{n}=108)$ quanto o médico residente $(82,6 \%, \mathrm{n}=124)$ todos os dias durante a internação. Quando perguntados sobre a formação acadêmica do médico residente, $43 \%(n=62)$ souberam responder de forma correta, identificando o residente como um médico em período de especialização em ortopedia e traumatologia. Dos $57 \%(n=82)$ que não compreendiam a real formação acadêmica desses profissionais, $34 \%(n=49)$ acreditavam que o médico residente era um médico ortopedista e traumatologista em início de carreira, $14 \%(n=20)$ acreditavam que o residente era um estudante de medicina e os $9 \%(n=13)$ restantes atribuíram outras definições a estes profissionais ou não souberam responder (-Tabela 2). 
Tabela 2 Avaliação das expectativas do paciente e avaliação do conhecimento sobre a formação do residente

\begin{tabular}{|c|c|}
\hline Respostas & $n(\%)$ \\
\hline $\begin{array}{l}\text { Espera encontrar o cirurgião principal todos } \\
\text { os dias durante a internação }\end{array}$ & $\mathrm{n}=150$ \\
\hline Sim & $108(72 \%)$ \\
\hline Não & $42(28 \%)$ \\
\hline $\begin{array}{l}\text { Espera encontrar o médico residente todos } \\
\text { os dias durante a internação }\end{array}$ & $\mathrm{n}=150$ \\
\hline Sim & $124(82,6 \%)$ \\
\hline Não & $26(17,3 \%)$ \\
\hline $\begin{array}{l}\text { O que sabe a respeito da formação acadêmica } \\
\text { e profissional do médico residente em } \\
\text { ortopedia e traumatologia }\end{array}$ & $\mathrm{n}=144$ \\
\hline $\begin{array}{l}\text { Ainda é um estudante de medicina } \\
\text { em formação }\end{array}$ & $20(13,8 \%)$ \\
\hline $\begin{array}{l}\text { É um médico que está se especializando } \\
\text { em ortopedia e traumatologia }\end{array}$ & $62(43,0 \%)$ \\
\hline $\begin{array}{l}\text { É um médico especializado em ortopedia e } \\
\text { traumatologia em início de carreira }\end{array}$ & $49(34,0 \%)$ \\
\hline Outros & $13(9,0 \%)$ \\
\hline $\begin{array}{l}\text { Sabe identificar o cirurgião principal } \\
\text { responsável por seus cuidados }\end{array}$ & $\mathrm{n}=151$ \\
\hline Sim & $50(33,1 \%)$ \\
\hline Não & $101(66,2 \%)$ \\
\hline $\begin{array}{l}\text { Sabe identificar o médico residente } \\
\text { envolvido em seus cuidados }\end{array}$ & $\mathrm{n}=147$ \\
\hline Sim & $21(14,3 \%)$ \\
\hline Não & $126(85,7 \%)$ \\
\hline
\end{tabular}

Os participantes também foram questionados quanto à sua percepção em relação à participação do médico residente em diferentes etapas do seu tratamento. Em relação à consulta ambulatorial, 43,3\% $(n=65)$ disseram preferir que o atendimento fosse realizado em conjunto pelo médico principal e o residente, enquanto apenas $17,3 \%(n=26)$ relataram se sentir seguros em ser atendidos apenas pelo residente neste momento. Já em relação ao atendimento na enfermaria, enquanto $39,3 \%(n=59)$ disseram preferir o atendimento em conjunto entre médico principal e o residente, $30,6 \%$ $(n=46)$ relataram se sentir seguros e confortáveis em serem assistidos apenas pelo médico residente. A avaliação da percepção dos pacientes em relação aos cuidados dos médicos residentes no centro cirúrgico revelou que a maioria dos participantes $(61 \%, \mathrm{n}=91)$ prefeririam ser assistidos em conjunto pelo médico residente e o cirurgião principal, enquanto apenas $11 \%(n=16)$ dos estudados disseram se sentir seguros e confortáveis sendo assistidos exclusivamente por residentes, caso fosse permitido ( $\mathbf{- T a b e l a ~} \mathbf{3}$ ).

Por fim, 68,5\% $(n=109)$ dos participantes disseram acreditar que um maior número de médicos envolvidos nos seus cuidados poderia melhorar a qualidade da assistência oferecida. Quanto ao papel do residente na comunicação entre o cirurgião e o paciente, $80,2 \% \quad(n=118)$ afirmaram que o médico residente melhora esta comunicação, enquanto apenas $1,3 \%(n=2)$ disseram que a presença do residente piora a comunicação entre paciente e cirurgião. Quando indagados quanto à relação entre a presença de programas de residência e a atenção recebida durante a internação, 85,5\% (n=124) dos participantes relataram receber mais atenção em hospitais com programa de residência médica, $4,1 \%(n=6)$ declararam receber menos atenção em hospitais escola e $10,3 \%(n=15)$ não tiveram internações prévias em hospitais sem programa de residência médica ( - Tabela 4 ).

Não encontramos associação entre gênero, idade ou renda, e a sensação de conforto e segurança em ser atendido pelo residente. Contudo, esta análise revelou que a percepção de conforto quanto à atuação do residente variou em relação à etapa do atendimento e o nível de escolaridade dos pacientes, onde identificamos relação entre o menor nível de escolaridade e maior percepção de conforto em ser assistido apenas pelo médico residente nas consultas ambulatoriais $(p=0,04) \mathrm{e}$ na enfermaria $(p=0,03)$. Tal associação não foi observada em relação à atuação isolada do médico residente no ambiente cirúrgico, uma vez que, independentemente do nível de educação, os pacientes relataram requerer a presença do médico principal durante o procedimento $(p=0,27)$.

Tabela 3 Percepção do paciente sobre a participação do residente

\begin{tabular}{|c|c|c|c|c|c|c|c|c|}
\hline \multirow[b]{3}{*}{$\begin{array}{l}\text { Percepção do paciente sobre a } \\
\text { participação do residente }\end{array}$} & \multicolumn{8}{|c|}{ Resposta } \\
\hline & \multicolumn{2}{|c|}{$\begin{array}{l}\text { Seguro e } \\
\text { confortável } \\
\text { em ser } \\
\text { atendido } \\
\text { apenas pelo } \\
\text { residente }\end{array}$} & \multicolumn{2}{|c|}{$\begin{array}{l}\text { Prefere ser } \\
\text { atendimento } \\
\text { pelo médico } \\
\text { principal em } \\
\text { conjunto com } \\
\text { o residente }\end{array}$} & \multicolumn{2}{|c|}{$\begin{array}{l}\text { Prefere ser } \\
\text { atendido } \\
\text { apenas pelo } \\
\text { médico } \\
\text { principal }\end{array}$} & \multicolumn{2}{|c|}{$\begin{array}{l}\text { Não souberam } \\
\text { opinar }\end{array}$} \\
\hline & $\mathrm{n}$ & $\%$ & $\mathbf{n}$ & $\%$ & $\mathbf{n}$ & $\%$ & $\mathbf{n}$ & $\%$ \\
\hline Consulta ambulatorial & 26 & 17,3 & 65 & 43,3 & 17 & 11,3 & 42 & 28 \\
\hline Enfermaria & 46 & 30,6 & 59 & 39,3 & 9 & 6 & 36 & 24 \\
\hline Cirurgia & 16 & 10,7 & 91 & 61 & 14 & 9,4 & 28 & 18,7 \\
\hline
\end{tabular}


Tabela 4 Papel do residente na assistência ao paciente

\begin{tabular}{|l|l|}
\hline Respostas & $n$ (\%) \\
\hline $\begin{array}{l}\text { Acredita que o envolvimento de um } \\
\text { elevado número de médicos pode } \\
\text { melhorar a qualidade dos cuidados }\end{array}$ & $\mathrm{n}=150$ \\
\hline Sim & $109(68,5 \%)$ \\
\hline Não & $18(11,3 \%)$ \\
\hline Não tem opinião & $23(14,4 \%)$ \\
\hline $\begin{array}{l}\text { Papel do Residente na comunicação entre } \\
\text { o paciente e o cirurgião principal }\end{array}$ & $\mathrm{n}=147$ \\
\hline Melhora a comunicação & $118(80,2 \%)$ \\
\hline Piora a comunicação & $2(1,3 \%)$ \\
\hline Não altera a comunicação & $27(18,3 \%)$ \\
\hline $\begin{array}{l}\text { Em relação à atenção recebida em hospital } \\
\text { escola comparado a hospitais sem } \\
\text { programa de residência médica }\end{array}$ & $\mathrm{n}=145$ \\
\hline $\begin{array}{l}\text { Recebi mais atencão em hospitais com } \\
\text { programa de residência médica }\end{array}$ & $124(85,5 \%)$ \\
\hline $\begin{array}{l}\text { Recebi menos atencão em hospitais } \\
\text { com programa de residência médica }\end{array}$ & $6(4,1 \%)$ \\
\hline Sem internações prévias & $15(10,3 \%)$ \\
\hline
\end{tabular}

\section{Discussão}

No Brasil, a prática de sobreposição de cirurgias é bastante comum em hospitais acadêmicos, onde o cirurgião principal pode iniciar um novo procedimento em outra sala de cirurgia sem ter finalizado o primeiro, deixando a cargo do médico residente a conclusão das partes não críticas do procedimento. Nestes hospitais escola, a participação do médico residente nas diversas etapas da assistência prestada ao paciente é essencial para a sua formação; ${ }^{13}$ contudo, ainda não há estudos nacionais que visem compreender a visão do paciente sobre a atuação do médico residente durante seu tratamento.

O presente estudo evidencia que o médico residente tem papel importante na facilitação da comunicação entre o paciente e o cirurgião principal, e que, apesar de menos da metade dos participantes conhecerem sobre a formação acadêmica dos residentes, de uma forma geral, eles se mostraram seguros e confortáveis sendo assistidos por médicos residentes em companhia do cirurgião principal.

Chama atenção o fato de que, em nossa amostra, apenas $33 \%$ dos pacientes souberam identificar o cirurgião responsável por seu tratamento e um percentual ainda menor soube identificar o médico residente, ainda que não tenhamos conseguido avaliar se os pacientes conseguiam identificar o cirurgião ou o residente com precisão. Este achado difere dos resultados encontrados por Cowles et al., ${ }^{12}$ que realizaram estudo similar em um hospital acadêmico e encontraram que $86 \%$ dos pacientes sabiam identificar o médico encarregado do seu tratamento. Acreditamos que o número elevado de médicos envolvidos no tratamento dos pacientes, aliado à falta de conhecimento sobre a atuação do residente possam ter contribuído para essa confusão.

Nossos resultados mostram que a percepção de conforto e segurança com a participação do residente varia de acordo com a etapa do tratamento, sendo a atuação do residente, de forma isolada, bem aceita na enfermaria, onde, dentre os pacientes que tinham uma opinião sobre o tema, $\sim 40 \%$ relataram se sentir seguros em serem atendidos apenas pelo residente, mas não na consulta ambulatorial ou durante a cirurgia, onde os percentuais encontrados foram de $24 \%$ e $13 \%$, respectivamente. Outros estudos reportaram resultados semelhantes, ao evidenciarem que os pacientes se sentem confortáveis em serem assistidos pelo residente fora do ambiente cirúrgico, mas não durante a cirurgia. ${ }^{12,14}$ Apesar do resultado, é importante ressaltar que estudos prévios mostraram que a participação de residentes no procedimento cirúrgico, sejam eles gerais ou ortopédicos, não está associada a incrementos nas taxas de mortalidade ou complicações gerais, sendo segura para os pacientes. ${ }^{10,15,16}$

De forma interessante, quando os pacientes foram estratificados em relação à escolaridade, encontramos que aqueles que não haviam completado o ensino médio se sentiam mais confortáveis quanto a serem atendidos apenas pelo médico residente na consulta ambulatorial ou na enfermaria. Entretanto, não encontramos, dentre os fatores avaliados neste estudo, motivos que justificassem essa diferença.

Nossos resultados também evidenciam que, ainda que apenas uma pequena parcela dos pacientes sinta segurança em ser assistido apenas pelo residente no ambiente cirúrgico, $75 \%$ daqueles que tinham uma opinião formada sobre esta questão disseram preferir que o procedimento fosse realizado pelo cirurgião em conjunto com o médico residente, indicando uma disposição em contribuir para a formação técnica do residente, como já observado em outros estudos. ${ }^{12}$ Acreditamos que um dos motivos da preferência pela atuação conjunta do médico principal com o médico residente seja pelo fato de os participantes apontarem que a presença do médico residente facilita a comunicação entre médico e paciente, resultado similar ao encontrado por Cowles et al. ${ }^{12} \mathrm{O}$ papel do residente na comunicação pode ser fundamental para o estabelecimento da relação de confiança entre médico e paciente, fator que é fundamental para adesão e complacência ao tratamento e que se correlaciona positivamente com a satisfação do paciente com o tratamento. ${ }^{17}$

De uma forma geral, nossos resultados corroboram o estudo de Cowles ao encontrar que o ambiente acadêmico hospitalar é bem tolerado pelos pacientes, uma vez que a maioria dos participantes disse acreditar que o maior número de médicos pode melhorar a qualidade da assistência recebida e relataram ter recebido mais atenção durante a internação em hospitais com residência médica. ${ }^{12}$

Uma das principais limitações do presente estudo é o fato de ter sido realizado com pacientes de um único hospital de ensino e com pacientes atendidos por uma mesma especialidade ortopédica. Apesar disso, acreditamos que os resultados desses estudos possam ser extrapolados por refletirem percepções comuns aos pacientes, independentemente do tipo de tratamento a que serão submetidos. Dessa forma, 
nossos resultados são relevantes por trazerem à tona a visão do usuário do sistema de saúde público do país, suscitando assim discussões mais amplas sobre o papel do residente e a prática de sobreposição de cirurgias.

\section{Conclusão}

Embora parte dos usuários do SUS não compreendam com exatidão a real formação profissional do médico residente em ortopedia e traumatologia, a participação dos residentes, em conjunto com o médico responsável, é bem tolerada nas diversas etapas da assistência prestada aos pacientes. Nossos resultados colocam os residentes como importantes atores na relação médico-paciente e sugerem uma disposição dos pacientes em contribuir para a educação dos residentes, reforçando a missão dos hospitais de ensino de criar condições favoráveis para educação em saúde sem perder o foco na excelência da assistência prestada aos pacientes.

\section{Suporte Financeiro}

Não houve suporte financeiro de fontes públicas, comerciais, ou sem fins lucrativos.

Conflito de Interesses

Os autores declaram não haver conflito de interesses.

\section{Referências}

1 Suarez JC, Al-Mansoori AA, Borroto WJ, Villa JM, Patel PD. The Practice of Overlapping Surgery Is Safe in Total Knee and Hip Arthroplasty. JBJS Open Access 2018;3(03):e0004

2 Sun E, Mello MM, Rishel CA, et al. Multicenter Perioperative Outcomes Group (MPOG). Association of Overlapping Surgery With Perioperative Outcomes. JAMA 2019;321(08):762-772

3 Zhang AL, Sing DC, Dang DY, et al. Overlapping Surgery in the Ambulatory Orthopaedic Setting. J Bone Joint Surg Am 2016;98 (22):1859-1867

4 Abelson J, Saltzman J, Kowalczyk L, Allen S. Clash in the name of care [acesso em: 8 Nov 2018]. The Boston Globe 2015 Oct 25. Disponível em: https://apps.bostonglobe.com/spotlight/clash-inthe- name-of-care/story/
5 Edgington JP, Petravick ME, Idowu OA, Lee MJ, Shi LL. Preferably Not My Surgery: A Survey of Patient and Family Member Comfort with Concurrent and Overlapping Surgeries. J Bone Joint Surg Am 2017;99(22):1883-1887

6 Langerman A. Concurrent Surgery and Informed Consent. JAMA Surg 2016;151(07):601-602

7 Levin PE, Moon D, Payne DE. Overlapping and Concurrent Surgery: A Professional and Ethical Analysis. J Bone Joint Surg Am 2017;99(23):2045-2050

8 Ponce BA, Wills BW, Hudson PW, et al. Outcomes with overlapping surgery at a large academic medical center. Ann Surg 2019; 269(03):465-470

9 Hyder JA, Hanson KT, Storlie CB, et al. Safety of overlapping surgery at a high-volume referral center. Ann Surg 2017;265 (04):639-644

10 Edelstein AI, Lovecchio FC, Saha S, Hsu WK, Kim JY. Impact of Resident Involvement on Orthopaedic Surgery Outcomes: An Analysis of 30,628 Patients from the American College of Surgeons National Surgical Quality Improvement Program Database. J Bone Joint Surg Am 2014;96(15):e131

11 D'Souza N, Hashimoto DA, Gurusamy K, Aggarwal R. Comparative Outcomes of Resident vs Attending Performed Surgery: A Systematic Review and Meta-Analysis. J Surg Educ 2016;73(03): 391-399

12 Cowles RA, Moyer CA, Sonnad SS, et al. Doctor-patient communication in surgery: attitudes and expectations of general surgery patients about the involvement and education of surgical residents. J Am Coll Surg 2001;193(01):73-80

13 Beasley GM, Pappas TN, Kirk AD. Procedure Delegation by Attending Surgeons Performing Concurrent Operations in Academic Medical Centers: Balancing Safety and Efficiency. Ann Surg 2015; 261(06):1044-1045

14 Goh LW, Lim AY. Surgical training in Singapore: will patients consent to trainee surgeons performing their operations? Ann Acad Med Singapore 2007;36(12):995-1002

15 Itani KM, DePalma RG, Schifftner $T$, et al. Surgical resident supervision in the operating room and outcomes of care in Veterans Affairs hospitals. Am J Surg 2005;190(05):725-731

16 Jordan SW, Mioton LM, Smetona J, et al. Resident involvement and plastic surgery outcomes: an analysis of 10,356 patients from the American College of Surgeons National Surgical Quality Improvement Program database. Plast Reconstr Surg 2013;131(04): 763-773

17 Ha JF, Longnecker N. Doctor-patient communication: a review. Ochsner J 2010;10(01):38-43 
444 Atuação do médico residente em ortopedia: Qual a visão do usuário? Oliveira et al.

Annex 1 Formulário de Coleta de Dados

\begin{tabular}{|l|l|}
\hline INFORMAÇÕES DO PROJETO DE PESQUISA: \\
\hline Título: & $\begin{array}{l}\text { ATUAÇÃO DO MÉDICO RESIDENTE EM ORTOPEDIA E TRAUMATOLOGIA EM UM HOSPITAL } \\
\text { DE GRANDE PORTE DO SISTEMA ÚNICO DE SAÚDE. QUAL A VISÃO DO USUÁRIO? }\end{array}$ \\
\hline Pesquisador Responsável: & \\
\hline Centro ou Setor: & Centro de Atenção Especializada (CAE) TRAUMA \\
\hline
\end{tabular}

DADOS A COLETAR

Iniciais do participante:

Prontuário:

1) Idade:
( ) 18 a 35 anos
( ) 36 a 55 anos
( ) 56 a 75 anos
( ) acima de 76 anos

2) Sexo:
( ) Masculino
( ) Feminino

3) Nível de escolaridade:

( ) Ensino fundamental incompleto

( ) Ensino fundamental completo

( ) Ensino médio incompleto

( ) Ensino médio completo

( ) Ensino superior incompleto

( ) Ensino superior completo

4) Renda Mensal:
( ) até $\mathrm{R} \$ 1.499,99$
( ) entre $R \$ 1.500,00$ a $2.999,99$
( ) entre $R \$ 3.000,00$ a $4.999,99$
( ) entre $\mathrm{R} \$ 5.000,00$ a $9.999,99$
( ) acima de $\mathrm{R} \$ 10.000,00$

5) Trabalha como profissional de saúde?
( ) Sim
( ) Não

6) Possui algum parente de primeiro grau que atua como profissional de saúde?
( ) $\operatorname{Sim}$
( ) Não

7) Já foi submetido a algum procedimento cirúrgico antes desta internação?
( ) Sim
( ) Não

8) Você sabe quem é o cirurgião principal responsável pelos seus cuidados durante esta internação?

( ) Sim

( ) Não

Nome do médico:

9) Você espera encontrar o cirurgião principal todos os dias durante sua internação?

( ) Sim

( ) Não 
10) Você sabe quem são os médicos residentes envolvidos nos seus cuidados?

( ) $\mathrm{Sim}$

( ) Não

Nomes dos médicos:

11 ) Você espera encontrar os médicos residentes todos os dias durante sua internação?
( ) $\mathrm{Sim}$
( ) Não

12) O que você sabe a respeito da formação acadêmica e profissional do médico residente em ortopedia e traumatologia?

( ) Ainda é um estudante de medicina em formação

( ) É um médico que está se especializando em ortopedia e traumatologia

( ) É um médico especializado em ortopedia e traumatologia em início de carreira

( ) Outros:

13) Qual o seu nível de conforto e segurança sendo assistido por um médico residente em uma consulta médica?

( ) Me sinto seguro e confortável sendo atendido somente por um médico residente

( ) Prefiro que o atendimento no consultório seja feito em conjunto por um médico residente e pelo cirurgião principal

( ) Prefiro que o atendimento no consultório seja feito apenas pelo cirurgião principal

( ) Não tenho uma opinião formada sobre essa pergunta

14) Qual o seu nível de conforto e segurança sendo assistido por um médico residente em uma visita na enfermaria?

( ) Me sinto seguro e confortável sendo visitado somente por um médico residente

( ) Prefiro que a visita seja feito em conjunto por um médico residente e pelo cirurgião principal

( ) Prefiro que a visita seja feita apenas pelo cirurgião principal

( ) Não tenho uma opinião formada sobre essa pergunta

15) Qual o seu nível de conforto e segurança sendo operado por um médico residente?

( ) Me sinto seguro e confortável sendo operado somente por um médico residente

( ) Prefiro que o procedimento cirúrgico seja feito em conjunto por um médico residente e pelo cirurgião principal

( ) Prefiro que o procedimento cirúrgico seja feito apenas pelo cirurgião principal

( ) Não tenho uma opinião formada sobre essa pergunta

16) Você acredita que um elevado número de médicos envolvidos nos seus cuidados pode melhorar a qualidade da assistência médica?
( ) $\operatorname{Sim}$
( ) Não
( ) Não tenho uma opinião formada sobre essa pergunta

17) Qual o papel do médico residente na comunicação entre você e o cirurgião principal?

( ) Melhora a comunicação entre mim e meu cirurgião principal

( ) Piora a comunicação entre mim e meu cirurgião principal

( ) Não altera a comunicação entre mim e meu cirurgião principal

18) Com relação à atenção recebida pela equipe médica:

( ) Recebi mais atenção neste hospital quando comparado a internações prévias em outros hospitais sem programa de residência médica

( ) Recebi menos atenção neste hospital quando comparado a internações prévias em outros hospitais sem programa de residência médica

( ) Não tive internações prévias em outros hospitais sem programa de residência médica

19) Caso fosse permito por nossa legislação, você se sentiria seguro sendo operado por um médico residente sem a supervisão integral de um cirurgião mais experiente?

( ) Sim

( ) Não

( ) Não tenho uma opinião formada sobre essa pergunta

\begin{tabular}{|l|l|}
\hline Local e data & Assinatura e carimbo do pesquisador responsável pela coleta dos dados: \\
\hline Rio de Janeiro, __ de ___ de __ & \\
\hline
\end{tabular}

